\title{
Effect of COVID-19 on Access to Cancer Care in India
}

\author{
Nidhi Guptaª Awadhesh Pandey ${ }^{1} \quad$ Aarti Goyal $^{2}$ \\ ${ }^{1}$ Department of Radiation Oncology, Government Medical College \\ and Hospital, Chandigarh, Punjab, India \\ 2Department of Community Medicine and School of Public Health, \\ Post Graduate Institute of Medical Education and Research, \\ Chandigarh, Punjab, India
}

\section{South Asian J Cancer:2021;10:46-47.}

India's response to the COVID-19 pandemic has been very effective and strong. On March 24, 2020, India announced a total lockdown for 6 weeks, which was followed by 2 weeks of graded lockdown. ${ }^{1}$ Existing human resources and health care infrastructure was allocated to activities for COVID-19 response. ${ }^{2}$ Requirements of physical distancing and reluctance to visit health facility due to fears of being infected resulted in poor care-seeking for cancer patients. All of this led to problems of access for cancer treatment, which are usually delivered at regional cancer centers, most of which are located in big urban cities. We evaluated the impact of COVID-19 on utilization of cancer care services in a large tertiary care hospital in north India.

We obtained the data on the day-wise number of outpatient visits, patients treated using radiation therapy, and hospitalizations in the department of radiation oncology from a tertiary care public sector hospital in north India from March 1, 2019, to May 16, 2020. The data were analyzed using a segmented time-series regression analysis. The date of national lockdown, March 24, 2020, was considered as the point to differentiate the two parts of the time series. Auto regressive integrated moving average (ARIMA) and seasonal auto regressive integrated moving average (SARIMA) models were used to assess nonstationarity, nonseasonality, and autocorrelation, imbibed in the time series data. Finally, multiple linear regression was used to estimate change in the level and trend.

Prior to the onset of COVID-19 restrictions, 89 outpatients visited daily, 58 patients got radiation treatment, and 10 patients were hospitalized for cancer care per day. Following COVID-19 restrictions, the estimated number of OPD patients, patients receiving radiation therapy, and hospitalizations dropped abruptly by 66\% (58 patients), 91\% (53 patients) and 230\% (22 patients) per day, respectively - Fig. 1.

DOI https://doi.org/10.1055/s-0041-1733313 ISSN 2278-330X

How to cite this article: Gupta N, Pandey A, Goyal A, et al. Effect of COVID-19 on Access to Cancer Care in India South Asian J Cancer 2021;10(1):46-47.
Address for correspondence Nidhi Gupta, MD, DNB, Department of Radiation Oncology, Government Medical College and Hospital, Chandigarh, Punjab, India (e-mail: nidhiguptaonco@gmail.com).

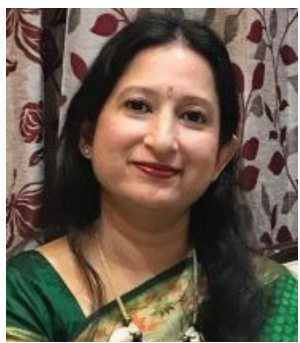

Dr Nidhi Gupta

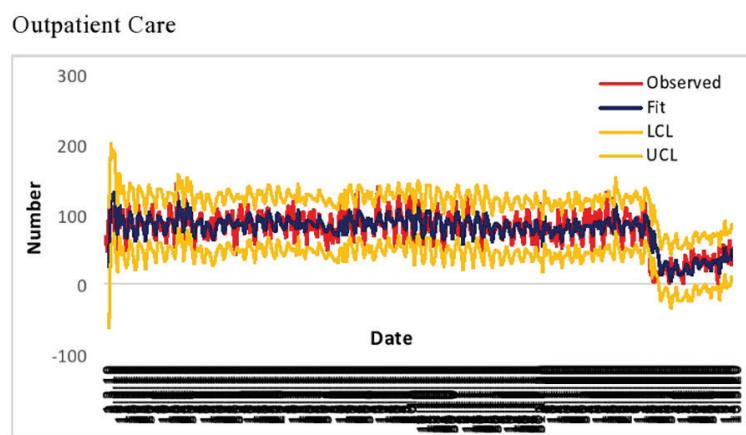

Hospitalization

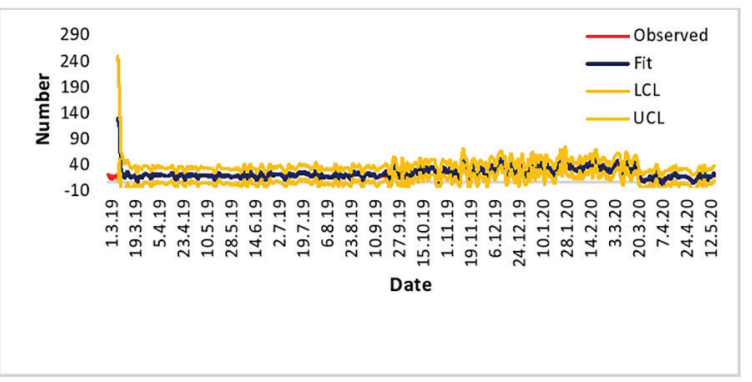

Radiation Therapy

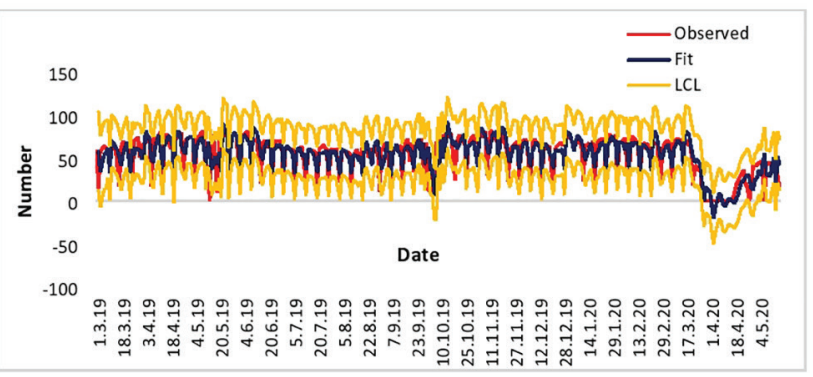

Fig. 1 Trends of outpatient consultation for cancer patients.

(c) 2021. MedIntel Services Pvt Ltd.

This is an open access article published by Thieme under the terms of the Creative Commons Attribution-NonDerivative-NonCommercial-License, permitting copying and reproduction so long as the original work is given appropriate credit. Contents may not be used for commercial purposes, or adapted, remixed, transformed or built upon. (https://creativecommons.org/licenses/by-nc-nd/4.0/).

Thieme Medical and Scientific Publishers Private Ltd A-12, Second Floor, Sector -2, NOIDA -201301, India 
The excessive drop in the indoor utilization can be explained by the doubling of the indoor bed strength, midway (august 2019) in pre-COVID period. The decline in number of female and male outpatient attendance was $79.5 \%$ and $51 \%$, respectively. This highlights the challenges faced by the females in access to health care services. The reduction of outpatient care for the various sites was highest for breast cancer $(100 \%)$, cervical cancer $(76 \%)$, and head and neck cancer (54\%).

Oncology services are marked by a continuity of treatment, where the aim of treatment is to add life years and better quality of life. Any significant gaps, interruptions, or delay in treatment impact locoregional control and survival. So, oncology centers across India should continue to provide services to cancer patients with utmost caution. All patients should be screened at the hospital entry points. Masks, thermometers, and hand rubs should be made available. Outpatient consultations through prior online appointment should be spaced, with cap on patient number to prevent crowding in the waiting area. Not more than one attendant should be allowed.

As the pandemic continues, health care systems must balance the need to provide necessary services while minimizing risk to patients and health care personnel. ${ }^{3}$ Personal protective equipment (PPE) should be made available to health care workers (HCW) involved in aerosol-generating procedures, and regular sanitization of the patient waiting areas, treatment areas and working areas should be done. All HCW should perform duties on a rotational basis, so that only a group of HCW are exposed during a particular period. ${ }^{4}$ In an event of a positive case within a cohort, it ensures that the other lot takes over and the service provision continues. Patient treatment should be prioritized based on the nature, stage, and expected outcome of the disease.

In the long term, learning from this pandemic should help us aim to modify the health delivery system, with more involvement of the primary health services who work in integration with tertiary care centers to provide continuity of care for chronic diseases. Increased use of technology and opening up telemedicine through transparent guidelines will improve access to health care even in remote locations in a geographically diverse country like India. ${ }^{5}$ The list of facilities allowed to administer chemotherapy remotely may be expanded. The district administration may coordinate with the primary health care team for providing referral transport on a periodic basis for easy movement of cancer patients to tertiary centers. Mobile medical units could be utilized on a regular basis for delivery of services, especially screening facilities, follow-ups, end of life/palliative care, and delivery of drugs. In view of the prolonged duration of pandemic, we should aim to ensure continuity of oncology services.

\section{Conflicts of Interest}

None declared.

\section{References}

1 COVID-19 lockdown in India. Available at: https://en.wikipedia.org/wiki/COVID-19_pandemic_lockdown_in_India. Accessed June 28, 2020

2 SOP for reallocation of residents/PG students and nursing students as part of hospital management of COVID. Available at: https://www.mohfw.gov.in/pdf/ COVID19SOPfordoctorsandnurses.pdfhttps://en.wikipedia org/wiki/COVID-19_pandemic_lockdown_in_India. Accessed June 28, 2020

3 Mehta P, Parikh P, Aggarwal S, et al. Has India met this enemybefore? From an eternal optimist's perspective: SARS-CoV-2. Indian J Med Sci2020;72:8-12 2020

4 Parikh P, Mehta P, Bansal S, et al. Protecting health-care professionals and workers (other than COVID-19 management facilities) from contamination during COVID-19 pandemic. Indian J Med Sci 2020;72:3-4

5 Telemedicine Practice Guidelines. Available at: https://www. mohfw.gov.in/pdf/Telemedicine.pdf. Accessed June 28, 2020 